\title{
TEMPORALLY EXTENDED ACTIONS FOR REINFORCEMENT LEARNING BASED SCHEDULERS
}

\author{
Prakhar Ojha ${ }^{1}$, Siddhartha R Thota ${ }^{2}$, Vani $^{1}{ }^{1}$, Mohit P Tahilianni ${ }^{1}$ \\ ${ }^{1}$ Department of Computer Engineering, National Institute of Technology Karnataka, \\ Surathkal, India \\ ${ }^{2}$ Department of Information Technology, National Institute of Technology Karnataka, \\ Surathkal, India
}

\begin{abstract}
Temporally extended actions have been proved to enhance the performance of reinforcement learning agents. The broader framework of 'Options' gives us a flexible way of representing such extended course of action in Markov decision processes. In this work we try to adapt options framework to model an operating system scheduler, which is expected not to allow processor stay idle if there is any process ready or waiting for its execution. A process is allowed to utilize CPU resources for a fixed quantum of time (timeslice) and subsequent context switch leads to considerable overhead. In this work we try to utilize the historical performances of a scheduler and try to reduce the number of redundant context switches. We propose a machine-learning module, based on temporally extended reinforcement-learning agent, to predict a better performing timeslice. We measure the importance of states, in option framework, by evaluating the impact of their absence and propose an algorithm to identify such checkpoint states. We present empirical evaluation of our approach in a maze-world navigation and their implications on "adaptive timeslice parameter" show efficient throughput time.
\end{abstract}

\section{KEYWORDS}

Temporal Extension of Actions, Options, Reinforcement Learning, Online Machine Learning, Operating System, Scheduler, Preemption

\section{INTRODUCTION}

One of the key reasons why humans can efficiently solve problems is their ability to create abstractions in complex world by ignoring irrelevant details. In case of artificial agents, there has been relatively lesser work on autonomously discovering useful abstractions. A system that can autonomously discover new abstractions can learn to act in more complex situations and deviate from its originally anticipated behaviour. In this work we want to extend the schedulers in operating systems by autonomously discovering useful abstractions. Scheduling is based on timesharing techniques where several processes are allowed to run "concurrently" so that the processor time is roughly divided into "slices", one for each runnable process. A single core processor, which can run only one process at any given instant, needs to be time multiplexed for running more processes simultaneously. Whenever a running process is not terminated upon exhausting its quantum time slice, a switch takes place where another process in brought into context. Linux processes have the capability of preemption [8]. If a process enters the running state, the kernel checks whether its priority is greater than the priority of the currently running process. If this condition is satisfied then the execution is interrupted and scheduler is invoked to select the process, which just became runnable. 
This type of time-sharing relies upon the interrupts and is transparent to processes. Otherwise, a process is also to be preempted when its time quantum expires. The duration, being critical for system performances, a natural question to ask would be - How long should a time quantum last? It should be neither too long nor too short [8]. Excessively short periods will cause system overhead because of large number of task switches. Consider a scenario where every task switch requires 10 milliseconds and the time slice is also set to 10 milliseconds, then at least $50 \%$ of the $\mathrm{CPU}$ cycles are being dedicated to task switch. On the other hand if quantum duration is too long, processes no longer appear to be executed concurrently [15]. For instance, if the quantum is set to five seconds, each runnable process makes progress for about five seconds, but then it stops for a very long time (typically, five seconds times the number of runnable processes). When a process has exhausted its time quantum, it is preempted and replaced by another runnable process. Every time a process is pushed out to bring in another process for execution (referred as context switch) several other elementary operations like swap-buffers, pipelines clearances, invalidate cache etc. take place making process switch a costly operation [16]. So preemption of a process leads to considerable overhead.

As there does not exist any direct relation between timeslice and other performance metrics, our work proposes a machine-learning module to predict a better performing timeslice. Even though the options framework gives convenient way to describe and reason with temporally extended actions, there is very little guidance regarding how good options can be found. The aim of this work is to address this issue by automatically finding sub-goal states for termination conditions of options. Most of the earlier works have considered frequency of visitation of states as prime heuristic to identify sub-goals [26]. The proposed adaptive time slice for preemption displays improvements in terms of the total time taken (Turnaround Time) after the submission of process to its completion, in-return creating more processor ticks for future. Most of present work has hard-wired classifiers that are applicable only to certain types of jobs. Having a reinforcementlearning agent with reward-function and temporally extended actions, which learns over time, gives the flexibility of adapting to dynamic systems. There is a primary assumption made in most of these works that the agent is restricted in the same environment but can confront with different tasks and that it can explore its environment sufficiently enough to gather necessary information. The subsequent sections will briefly discuss the fundamentals of reinforcement learning and options framework, which strives to continuously improve self by learning in any new environment.

The work presented in this paper is an extension to our previous work [18], where we only focused on applying primitive RL techniques and show their applicability in Linux schedulers. Here we aim at improving the adaptive module by means of incorporating temporal extensions in decision making. Our hope is that such extensions will remove lower level detailed action selection and allow for intelligent abstracted options. Following sections in this paper are organized as follows: Section 2 gives an overview of the related previous works and Section 3 explains the theory of reinforcement learning and options framework and sets up notation for this paper. Section 4 shows how we approach the problem in hand by proposing a novel design, integrate RL modules and run simulations and then followed by implementation details of knowledge base created and self-learning systems in Section 5. The results and analysis of our system's performance is evaluated in Section 6 followed by conclusions and discussions in Section 7. 


\section{RELATED WORKS}

Below section briefly discusses earlier works in relevant fields by applying machine learning techniques to CPU resources and Operating system parameters. Attempts have been made to use historical-data and learn the timeslice parameter, which judges the preemption time for a given process, and make it more adaptive.

To remember the previous execution behaviour of certain well-known programs, [10] studies the process times of programs in various similarity states. The knowledge of the program flow sequence (PFS), which characterizes the process execution behaviour, is used to extend the CPU time slice of a process. They also use thresholding techniques by scaling some feature to determine the time limit for context switching. Their experimental results show that overall processing time is reduced for known programs. Works related to Thread schedulers on multi core systems, using Reinforcement learning, assigns threads to different CPU cores [6], made a case that a scheduler must balance between three objectives: optimal performance, fair CPU sharing and balanced core assignment. They also showed that unbalanced core assignment results in performance jitter and inconsistent priority enforcement. A simple fix that eliminates jitter and presents a scheduling framework that balances these three objectives by algorithm based on reinforcement learning was explored.

For temporally actions, earlier work by Stolle et al. considered the frequency of visitationof states to identify subgoals [25]. For the room-hallway environment the main notion is to detect the hallway regions from the state space as they form the bottleneck for most paths from start to end state. The work in [7] has addressed scheduler problem based on making fixed classifiers over hand picked features. Here timeslice values were tried against several combination of attributes and patterns emerged for chosing better heuristic. However, their approach was compatible to only few common processes like random number generation, sorting etc. and unlike our work, not universally adaptive for any application. Reward based algorithms and their use in resolving the lock contention has been considered as scheduling problem in some the earlier works[2]. These hierarchal spin-locks are developed and priority assigned to processes to schedule the criticalsection access.Application run times are predicted using historical information in [1]. They derive predictions for run times of parallel applications from the run times of similar applications that have executed in the past. They use some of the following characteristics to define similarity: user, queue, load leveler script, arguments, network adapter, number of nodes, maximum run time, submission time, start time, run time. These characteristics are used to make a template which can find the similarity by matching. They use genetic algorithms (GA), which are are well known for exploring large search spaces, for identifying good templates for a particular workload. Statistical Regression methods, which work well on numeric data but not over nominal data, are used for prediction [5].

An application signature model for predicting performance is proposed in [4] over a given grid of resources. It presents a general methodology for online scheduling of parallel jobs onto multiprocessor servers, in a soft real-time environment. This model introduces the notion of application intrinsic behaviour to separate the performance effects of the runtime system from the behaviour inherent in the application itself. Reinforcement Learning is used for tuning its own value function that predicts the average future utility per time step obtained from completed jobs based on the dynamically observed state information. From this brief review of related literature, we draw the following conclusions: 
- It is possible to profitably predict the scheduling behaviour of programs. Due to the varied results in all above discussed works, we believe that the success of the approach depends upon the ML technique used to train on previous programs execution behaviour.

- A suitable characterization of the program attributes (features) is necessary for these automated machine learning techniques to succeed in prediction.

In specific to using reinforcement learning in realms of scheduling algorithms, most of the work is concentrated around ordering the processes like to learn better permutations of given list of processes, unlike our work of parameter estimation.

\section{FRAMEWORK}

\subsection{Reinforcement Learning}

Reinforcement learning (RL) is a collection of methods for approximating optimal solutions to stochastic sequential decision problems [6]. An RL system does not require a teacher to specify correct actions, instead, it tries different actions and observes their consequences to determine which actions are best. More specifically, in any RL framework, a learning agent interacts with its environment over a series of discrete time steps $t=0,1,2,3$. . Refer Figure.1. At each time $t$, the agent observes the environment state $s_{t}$, and chooses an action $a_{t}$, which causes the environment to transition to a new state $s_{t+1}$, and to reward the agent with $r_{t+1}$. In a Markovian system, the next state and reward depend only on the current state and present action taken, in a stochastic manner. To clarify notation used below, in a system with discrete number of states, $S$ is the set of states. Likewise, $A$ is the set of all possible actions and $A(s)$ is the set of actions available in states. The objective of the agent is to learn to maximize the expected value of reward received over time. It does this by learning a (possibly stochastic) mapping from states to actions called a policy. More precisely, the objective is to choose each action at so as to maximize the expected return $R$, given by,

$$
R:=E\left(\sum_{i=0}^{\infty} \gamma^{i} r_{t+i+1}\right)
$$

where $\gamma$ is the discount-rate parameter in range $[0,1]$, which allows the agent to trade-off between the immediate reward and future possible rewards.

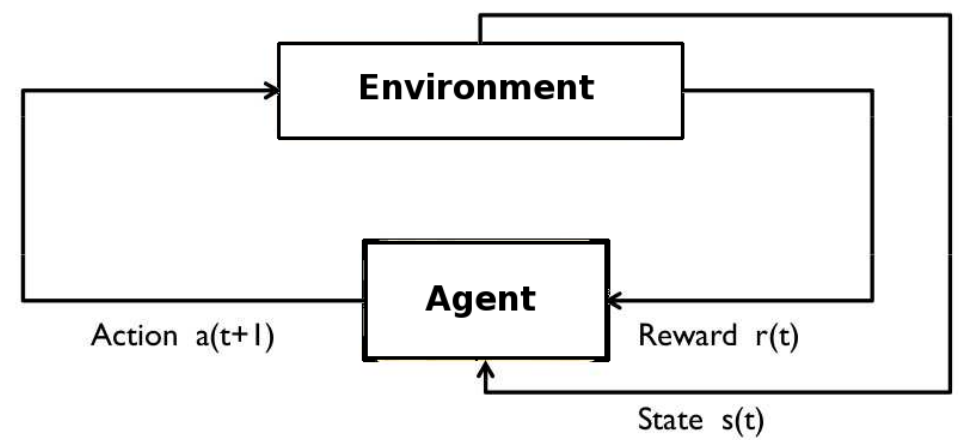

Fig.1 Concept of Reinforcement learning depicting iteraction between agent and environment 
Two common solution strategies for learning an optimal policy are to approximate the optimal value function, $\mathrm{V}^{*}$, or the optimal action-value function, $\mathrm{Q}^{*}$. The optimal value function maps each state to the maximum expected return that can be obtained starting in that state and thereafter always taking the best actions. With the optimal value function and knowledge of the probable consequences of each action from each state, the agent can choose an optimal policy. For control problems where the consequences of each action are not necessarily known, a related strategy is to approximate $\mathrm{Q}^{*}$, which maps each state and action to the maximum expected return starting from the given state, assuming that the specified action is taken, and that optimal actions are chosen thereafter. Both $\mathrm{V}^{*}$ and $\mathrm{Q}^{*}$ can be defined using Bellman -equations as

$$
Q^{*}(s, a)=\sum_{s^{\prime} \in S} P_{s s^{\prime}}^{a}\left[R_{s s^{\prime}}^{a}+\gamma \max _{a \prime \in A\left(s^{\prime}\right)} Q^{*}\left(s^{\prime}, a^{\prime}\right)\right]
$$

where s' is the state at next time step, $\mathrm{P}^{\mathrm{a}} \mathrm{ss}^{\prime}$ is its probability of transission and $\mathrm{R}_{\mathrm{ss}}^{\mathrm{a}}$ is the associated reward.

\subsection{Temporally Extended Actions: Options}

Options generalize trivial tasks into a single extended course of actions $[19,28]$. These temporally extended actions when selected by the agent, execute until a termination condition is met. During its execution, the actions of particular option are chosen according to its own policy. One can consider options as traditional open-loop-macros that can also follow a closed-loop policy in reaction to the environment. It is well established that by augmenting the agent's set of primitive actions with options, the agent's performance can be enhanced. This framework consists of an initiation set, a policy and termination condition $(I, \pi, \beta)$. An option can start in a state only if it is included in $\{\mathrm{I}\}$. Subsequently after selecting an option, the actions are chosen according to the policy $\pi$ until the option terminates stochastically according to $ß$. When the option terminates, the agent gets opportunity to select another option or primitive action based on initiation set. SemiMarkov decision process (SMDP) combines the above idea of a fixed set of options with MDPs. The optimal policy over a set of options $\mathrm{O}$ is addressed by SMDP version of one-step Q-learning learning methods.

\section{OUR APPROACH}

\subsection{Problem formulation}

Our prime motivation is to reduce the redundant preemptions that current schedulers do not take into account. To explain using a simple example, suppose a process has a very little burst time left and it is swapped due to the completion of its timeslice ticks, then the overhead of cacheinvalidation, pipeline clearing, context switching etc. reduces the efficiency. Hence having a flexible timeslice window will prevent the above scenario. This would also improve the total time taken after the submission of process to its completion, in return creating more processor ticks for future.

In this paper, we want to study the application of machine learning in operating systems and build learning modules so as to make the timeslice parameter flexible and adaptive. Our aim is to maintain the generality of our program so that it can be employed and learned in any environment. We also want to analyze how long it takes for a module to learn from its own experiences so that it can be usefully harnessed to save time. Our main approach is to employ reinforcement learning techniques for addressing this issue of continuous improvement. We want 
to formulate our learning through the reward-function which can self-evaluate its performance and improve overtime.

\subsection{Module Design}

Figure.2 gives an over all view of our entire system. It describes how our reinforcement learning agent makes use of the patterns learned initially and later on after having enough experiences it develops a policy of itself to use the prior history and reward-function.

Formally, these below steps capture the important end-to-end flow mechanism.

1. Program $X$ passes its requirements in user-space for acquiring resources from computer hardware. These requirements are received by our agent.

2. Reinforcement learning agent uses its knowledge base to make decision. It uses patterns recognized in the initial stages to have a kick start with reasonable values and not random values. Later on knowledge base develops its history and reward function after sufficient number of experiences.

3. The information is passed from the user-space to kernel-space via a system call which will have to be coded by us. This kernel call will redirect the resource request to our modified scheduler.

4. The number of ticks to be allocated is found in the fields of new_timeslice and forwarded to CPU. And finally, CPU allocates these received orders in form of new ticks.

5 .

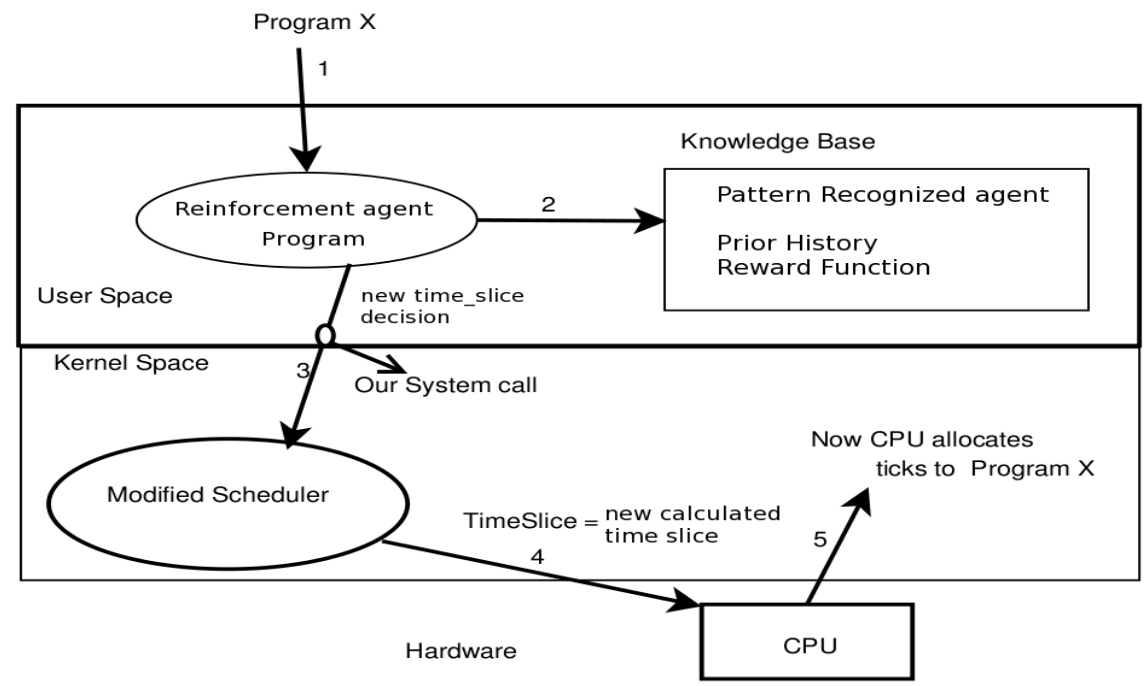

Fig.2 Bird's eye view of our design and implementation pipeline

As the intermediate system call and modified scheduler are the only changes required in the existing systems, we provide complete abstraction to the CPU and user-space.

\subsection{Modelling an RL agent}

We present here a model to simulate and understand the Reinforcement learning concepts and understand the updates of Bellman equation in greater depth [6]. We have created this software with an aim to visualize the results of changing certain parameters of RL functions and as a precursor for modelling scheduler. 


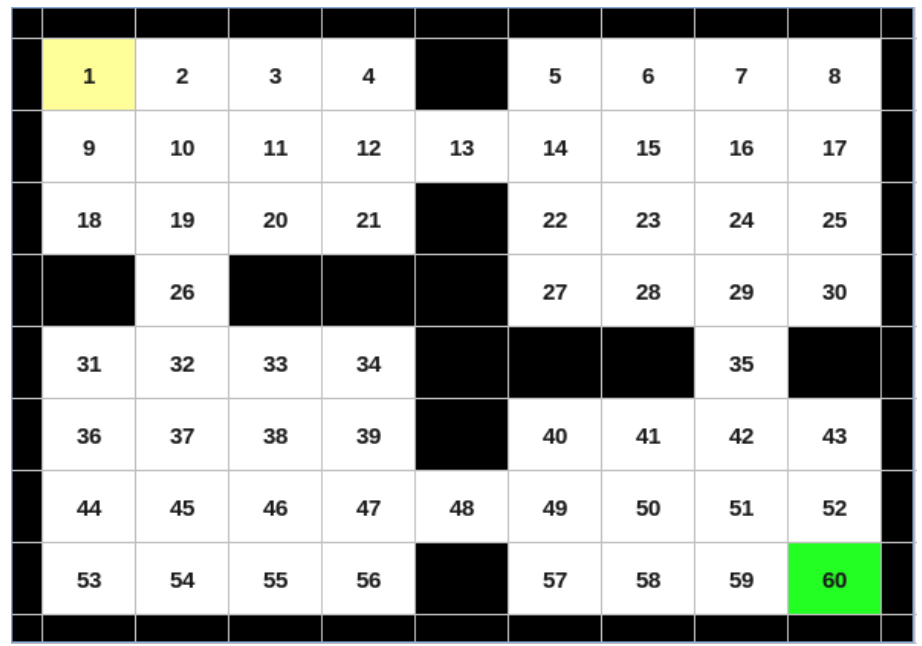

Fig.3 Maze showing the environment in which RL-agent interacts.

- Work-Space: Checkerboard setup with a grid like maze.

- Aim: To design an agent which finds its own way from the start state to goal state. The agent is designed to explore the possible paths from start state and arrive at goal state. Each state has four possible actions N, S, E \& W. Collision with wall has no effect.

- Description: Figure.3 depicts the maze which consists of rooms and walls. The whole arena is broken into states. Walls are depicted by dark-black solid blocks denoting that the agent cannot occupy these positions. The other blocks are number $1,2,3 \ldots . .60$ as the possible states in which agent can be. Agent is situated at $S_{1}$ at time $t=0$ and at every future action it tries to find its way to the goal state $S_{60}$.

- Reward-function: Transition to goal state gives a reward of +100 . Living penalty is 0 . Hence the agent can take as long time as it wants to learn the optimal policy. This parameter will be changed in case of real time schedulers. Reward Updating policy has Temporal difference updates with learning rate (alpha) $=0.2$

Initially the agent is not aware of its environment and explores it to find out. Later it learns a policy to make that wise decision about its path finding. Code (made publicly available) is written in $\mathrm{C}$ language for faster excution time and the output is an HTML file to help better visualize the reward updates and policy learned. Results and policies learned will be described in later sections.

\subsection{Identifying Options}

Our algorithm is based on the intuition that removal of different states (blocking them) from the state-space causes different amounts of changes in the cumulative rewards of neighboring states. We draw analogy from Cooperative Game Theory, wherein a player gains importance based on his individual contribution to the group as well as his collaborative efforts with every other member (or subset) of the group [24]. Based on this idea, shapley value gives a fair share of group-reward to any player based on analyzing the difference in group's performance with and without the specific player. We are incorporating this concept into state-space domain by blocking a particular state and calculating the net variance of difference in cumulative rewards of its neighboring states. Upon specifying initiation set and learning internal policies of options 
using standard RL methods, we focus to selecting sub-goal states that can potentially be checkpoints. In order to learn future options, the agent starts to explore the environment ahead of time by means of solving random tasks. During this exploration, agent gathers statistics of learning a policy on complete state-space and learning a policy after blocking one of the random states from state-space. This helps in calculating level of influence on its neighboring states upon removal. The below algorithm gives a step wise guidance to our approach, followed by experimental details.

\section{Algorithm}

1. Select a few random tasks by choosing pairs $\{\mathrm{S}, \mathrm{T}\}$ uniformly over start and target states.

2. For each task

a. Perform standard Q-learning to obtain values of states for going from $\mathrm{S}$ to $\mathrm{T}$.

b. Repeat the earlier Q-learning task by blocking states from state-space systematically.

3. For every state, calculate the difference in cumulative rewards of its neighbors when it was and was not blocked. Calculate the average variance to avoid any bias due to unequal number of neighbors.

4. Choose the checkpoint states by observing local maxima in variance values amongst neighboring states.

Blocking a state refers to no possible transition either from or to this state. An option can be formulated in such a way that checkpoint states are included under termination condition and we can further use SMDP Q-learning in order to learn a policy over these identified options.

\subsection{Simulation}

As the scheduler resides deep in the kernel, measuring the efficacy of scheduling policies in Linux is difficult. Tracing can actually change the behavior of scheduler and hide defects or inefficiencies. For example, an invalid memory reference in the scheduler will almost certainly crash the system [8]. Debugging information is limited and not easily obtained or understood by new developer. This combination of long crash-reboot cycles and limited debugging information can result in a time-consuming development process. Hence we resort to a good simulator of the Linux scheduler that we can manipulate for verifying our experiments instead of changing kernel directly.

\section{LinSched: Linux Scheduler simulation}

LinSched is a Linux scheduler simulator that resides in user space [11]. It isolates the scheduler subsystem and builds enough of the kernel environment around it that it can be executed within user space. Its behaviour can be understood by collecting relevant data through a set of available APIs. Its wrappers and simulation engine source is actually a Linux distribution. As LinSched uses the Linux scheduler within its simulation, it is much simpler to make changes, and then integrate them back into the kernel.

We would like to mention few of the essential simulator side APIs below, which we experimented over. One can utilize them to emulate the system calls and program the tasks. They 
are used to test any policy that are under development and see the results beforehand implementing at kernel directly. linsched_create_RTrr(...) -creates a normal task and sets the scheduling policy to normal. void linsched_run_sim(...) -begins a simulation. It accepts as its only argument the number of ticks to run. At each tick, the potential for a scheduling decision is made and returns when it is complete. Few statistics commands like void linsched_print_task_stats() and void linsched_print_group_stats() give more detailed analysis about a task we use. We can find the total execution time for the task (with task_exec_time(task)), time spent not running (task->sched_info.run_delay), and the number of times the scheduler invoked the task (task$>$ sched_info.pcount).

We conducted several experiments over the simulator on normal batch of jobs by supplying it work load in terms of process creation. First 2 normal tasks are created with no difference and ambiguity (using linsched_create_normal_task(...)). We next created a job which runs on normal scheduler and has a higher priority by assigning nice value as -5 . Similarly we experimented with jobs which had lower priority of +5 , followed by populating another normal and neutral priority. On the other hand, we also verified our experiments over batch tasks which are created with low and high priorities. They are all computation intensive tasks which run in blocks or batches. (using linsched_create_batch_task(...)). And then finally one real-time FIFO task with priority varying in range of 50-90, and one round-robin real-time task with similar priority range. Each task as created is assigned with task_id which is realistic as in real linux machines. Initially all tasks are created one after other and then after scheduler_tick() function times out, it is called for taking decision on other processes in waiting/ready queue. The relevant results will be discussed in subsequent sections.

\section{IMPLEMENTATION AND EXPERIMENTS}

\subsection{Knowledge Base Creation}

\subsubsection{Creating Dataset}

To characterize the program execution behaviour, we needed to find the static and dynamic characteristics. We used readelf and size commands to get the attributes. We built the data set of approximately 80 execution instances of five programs: matrix multiplication, quick sort, merge sort, heap sort and a recursive Fibonacci number generator. For instance, a script ran matrix multiplication program of size $700 \times 700$ multiple times with different nice values and selected the special time slice (STS), which gave minimum Turn Around Time (TaT). After collecting the data for the above programs with different input sizes, all of them were mapped to the best priority value. Data of the above 84 instances of the five programs were then classified into 11 categories based on the attribute time slice classes with each class having an interval of 50 ticks. We mapped the variance of timeslice against total Turnaround Time (TaT) taken by various processes like Insertion sort, Merge sort, Quick sort, Heap sorts and Matrix multiplication with input ranging from 1e4, 1e5, 1e6 after experimenting against all possible timeslices.

\subsubsection{Processing Dataset}

After extracting the features from executable filles, by readelf and size commands, we refine the number of attributes to only those few essential features which actually help in taking decision. A few significant deciding features which were later used for building decision tree are: RoData (read only data), Hash (size of hash table), Bss (size of uninitialized memory), DynSym (size of dynamic linking table), StrTab (size of string table). The less varying / non-deciding features are 
discarded. The best ranked special time slices to each instance to gauge were classified to the corresponding output of decision tree. The processed result was further fed as input to to the classifier algorithm (decision trees in our case) to build iterative if-else condition.

\subsubsection{Classification of Data}

To handle new incoming we have built a classifier with attributes obtained from previous steps. Decision tree rules are generated as the output from classification algorithm. We used WEKA (Knowledge analysis tool) to model these classifiers. Most important identified features are RoData, Bss and Hash . Finally groups are classified into 20 classes in ranges of timeslice. Few instances for Decision Tree Rules are mentioned below.

- if $\{($ RoData $<=72)$ AND (bss $<=36000032)$ AND (bss $<=4800032)$ AND (bss $<=3200032)\}$ then class $=13$

- if $\{($ RoData $<=72)$ AND (bss $<=36000032)$ AND (bss $<=$ 4800032) AND (bss > 3200032) $\}$ then class $=\mathbf{2}$

- if $\{($ RoData $<=72)$ AND (bss $<=36000032)$ AND (bss > 4800032) AND (bss $<=$ 7300032) $\}$ then class $=5$

- if $\{($ RoData $<=72)$ AND (bss $<=36000032)$ AND (bss > 4800032) AND (bss > 7300032) AND (bss $<=2000032)$ \} then class $=\mathbf{3}$

- if $\{($ RoData $<=72)$ AND (bss $>36000032)$ AND (bss $<=4800032)\}$ then class $=7$

- if $\{($ RoData $<=72)$ AND (bss $<=36000032)$ AND (bss $>4800032)$ AND (bss $>7300032)$ AND (bss $>2000032)\}$ then class $=\mathbf{0}$

- if $\{($ RoData $<=72)$ AND (bss $>36000032)$ AND (bss $<=4800032)\}$ then class $=4$

To give a better visualization of out features, we present in Table.l various statistics obtained for Heap sort with input size $3 \mathrm{e} 5$ and priority (nice value) set to 4 . These statistics help us decide the lowest Turnaround Time and lowest number of swaps taken for best priority class.

\begin{tabular}{|l|l||l|l|}
\hline Feature Name & Value & Feature Name & Value \\
\hline User time (seconds) & 0.37 & Voluntary context switches & 1 \\
\hline Minor (reclaiming frame) page faults & 743 & Involuntary context switches & 41 \\
\hline Percent of CPU this job got & $98 \%$ & File system outputs & 8 \\
\hline Elapsed (wall clock) time (h:mm:ss) & $0: 00.38$ & Socket messages sent & 0 \\
\hline Maximum resident set size (kbytes) & 1632 & Socket messages received & 0 \\
\hline Signals delivered & 0 & Page size (bytes) & 4096 \\
\hline
\end{tabular}

Table.1 Statistics obtained for Heap sort with input size 3e5 showing the classifier features.

\subsection{Self-Improving Module}

The self-learning module that is based on Reinforcement learning technique is proved to improve over time with its experience until converged to saturation. The input to this module is the group decision from the knowledge base in the previous step as the output of the if-else clause. Further, reinforcement learning module may give a new class if it decides from its policy learned over time of several running experiences. In the background this self improving module would explore for new classes which it could assign to a new incoming process. We modelled the scheduler actions as a markov decision process where decisions for assigning a new time slice solely based over current state and it need not have to take into account of the previous decisions. The policy mapping for states and their aggregate reward associated is done using the Bellman equations 


$$
V^{*}(s)=\max _{a \in A} \sum_{s^{\prime} \in S} P_{s s^{\prime}}^{a}\left[R_{s s^{\prime}}^{a}+\gamma V^{*}\left(s^{\prime}\right)\right]
$$

Figure.4 shows how using an array implementation of Doubly Linked List, we generated the above module. Temporal difference (TD method) was used for updating the reward-function with experiences and time. The sense of reward for the scheduler agent was set to be a function of inverse of waiting time of the process. The choice of such a reward function was to avoid the bias introduced by the inverse of total turnaround time (TaT), which is the least where compared to waiting time. This is because TaT is also inclusive of total number of swaps which in turn is dependent over the size of input and size of text, whereas waiting time does not depend over the size of input. We set the exploration vs. exploitation constant to be 0.2 which is still flexible under temperature coefficient mentioned above.

Input to this module is the class decision from knowledge base obtained in the previous step which is the output of the decision tree. It outputs a new class which RL module decides from its policy generated over time of running. Reward sense is given by the inverse of waiting time of the process. We have used exploration vs. exploitation $\varepsilon$-greedy constant as 0.2 .

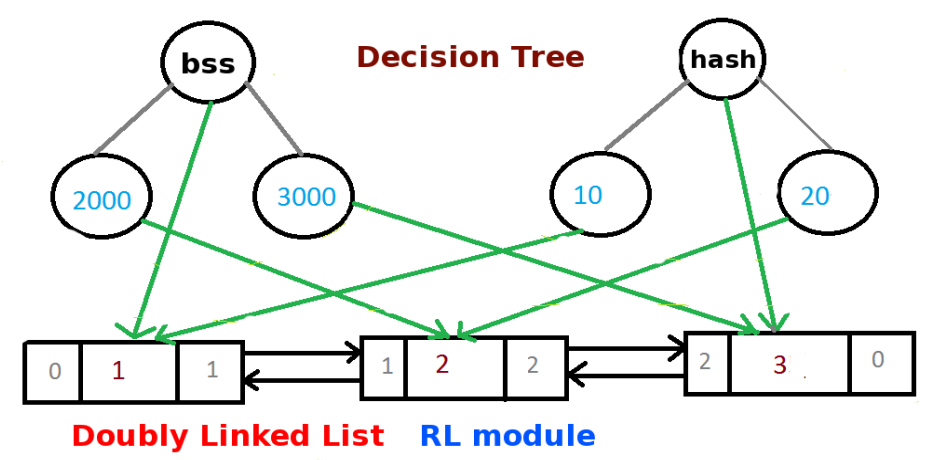

Fig.4 Integration of self learning module with decision tree knowledge base.

In our experimental Setup, we used WEKA (Knowledge analysis tool) for Decision trees and attribute selection. For compilation of all programs we used gcc (GNU_GCC) 4.5.1 (RedHat 4.5.1-4). To extract the attributes from executable/binary we used readelf $\&$ size command tools. For graph plots and mathematical calculations we used Octave.

\subsection{Temporal Extension}

We chose maze-world navigation task, as shown in Figure.3, to validate our hypothesis, where the setup can be perceived as rooms connected via hallways. The rooms-hallway environment is broken into 60 states where an agent can occupy only one state at any point of time. The agent tries to find the goal state by traversing a path in the state-space. There are four stochastic primitive actions that can move the agent North, South, East and West. Each action moves the agent in expected direction with probability 0.8 and one of the other 3 directions with probability 0.2 , chosen randomly. If the agent attempts to move into a blocked state or wall, it stays in the same position. The agent starting at state: 1 with no penalty being incurred for living or hitting the walls. The discount factor for future rewards is 0.7 and there are no associated transition rewards anywhere else except for exiting the maze at goal state:60. This environment is depicted in Figure.3. We try to address the problem of finding bottleneck regions by treating them as multiple-instance learning problem [20] with diverse density [21]. Here the system attempts to 
identify a target concept on the basis of paths of positive and negative instances. A positive path has at least one successful instance while negative path consist of all failed attempts to reach goal. We place more importance to a negative bag that consists of observations made over an unsuccessful path trajectory. Identifying bottleneck regions is well fit by this paradigm.

\section{RESULTS AND ANALYSIS}

For the maze environment as shown in Figure.3, one can intuitively expect the hallway regions to be obvious candidates for option termination state $B$. Our proposed algorithm indeed identifies those particular hallway states to be important and marks them as checkpoints. As any path crossing from one room to another needs to utilize the in-between hallway, blocking that particular hallway would require the agent to identify another roundabout way. Blocking a state that is well situated inside a room does not impact the cumulative rewards of its neighboring states.

\begin{tabular}{|c|c|c|c|c|c|c|c|c|}
\hline $\begin{array}{c}\text { Start } \\
1\end{array}$ & $\begin{array}{c}0.044403 \\
2\end{array}$ & $\begin{array}{c}-0.044382 \\
3\end{array}$ & $\begin{array}{c}-0.044366 \\
4\end{array}$ & & $\begin{array}{c}-0.044121 \\
5\end{array}$ & $\begin{array}{c}-0.04377 \\
6\end{array}$ & $\begin{array}{c}-0.043366 \\
7\end{array}$ & $\begin{array}{c}-0.043689 \\
\mathbf{8}\end{array}$ \\
\hline $\begin{array}{c}-0.044398 \\
9\end{array}$ & $\begin{array}{c}-0.044388 \\
10\end{array}$ & $\begin{array}{c}-0.044366 \\
\mathbf{1 1}\end{array}$ & $\begin{array}{c}-0.035496 \\
12\end{array}$ & $\begin{array}{c}-0.022771 \\
13\end{array}$ & $\begin{array}{c}-0.016785 \\
14\end{array}$ & $\begin{array}{c}-0.04331 \\
15\end{array}$ & $\begin{array}{c}-0.039548 \\
16\end{array}$ & $\begin{array}{c}-0.043095 \\
17\end{array}$ \\
\hline $\begin{array}{c}-0.044393 \\
18\end{array}$ & $\begin{array}{c}-0.042062 \\
19\end{array}$ & $\begin{array}{c}-0.044379 \\
\mathbf{2 0}\end{array}$ & $\begin{array}{c}-0.044366 \\
\mathbf{2 1}\end{array}$ & & $\begin{array}{c}-0.04326 \\
22\end{array}$ & $\begin{array}{c}-0.042155 \\
23\end{array}$ & $\begin{array}{c}-0.034424 \\
\mathbf{2 4}\end{array}$ & $\begin{array}{c}-0.041717 \\
25\end{array}$ \\
\hline & $\begin{array}{c}-0.039307 \\
\mathbf{2 6}\end{array}$ & & & & $\begin{array}{c}-0.042155 \\
27\end{array}$ & $\begin{array}{c}-0.032944 \\
\mathbf{2 8}\end{array}$ & $\begin{array}{c}0.157096 \\
29\end{array}$ & $\begin{array}{c}-0.040569 \\
\mathbf{3 0}\end{array}$ \\
\hline $\begin{array}{c}-0.044273 \\
31\end{array}$ & $\begin{array}{c}-0.037991 \\
32\end{array}$ & $\begin{array}{c}-0.043851 \\
33\end{array}$ & $\begin{array}{c}-0.04331 \\
\mathbf{3 4}\end{array}$ & & & & $\begin{array}{c}0.166105 \\
35\end{array}$ & \\
\hline $\begin{array}{c}-0.044101 \\
36\end{array}$ & $\begin{array}{c}-0.043966 \\
37\end{array}$ & $\begin{array}{c}-0.04331 \\
\mathbf{3 8}\end{array}$ & $\begin{array}{c}-0.038796 \\
39\end{array}$ & & $\begin{array}{c}-0.033873 \\
\mathbf{4 0}\end{array}$ & $\begin{array}{c}-0.024322 \\
\mathbf{4 1}\end{array}$ & $\begin{array}{c}0.236145 \\
42\end{array}$ & $\begin{array}{c}0.022363 \\
43\end{array}$ \\
\hline $\begin{array}{c}-0.043903 \\
44\end{array}$ & $\begin{array}{c}-0.042032 \\
45\end{array}$ & $\begin{array}{c}-0.039522 \\
46\end{array}$ & $\begin{array}{c}0.052337 \\
47\end{array}$ & $\begin{array}{c}0.057534 \\
48\end{array}$ & $\begin{array}{c}0.109959 \\
49\end{array}$ & $\begin{array}{c}-0.005151 \\
\mathbf{5 0}\end{array}$ & $\begin{array}{c}0.035719 \\
\mathbf{5 1}\end{array}$ & $\begin{array}{c}0.405773 \\
52\end{array}$ \\
\hline $\begin{array}{c}-0.044061 \\
53\end{array}$ & $\begin{array}{c}-0.04377 \\
54\end{array}$ & $\begin{array}{c}-0.043095 \\
55\end{array}$ & $\begin{array}{c}-0.042532 \\
\mathbf{5 6}\end{array}$ & & $\begin{array}{c}-0.011696 \\
57\end{array}$ & $\begin{array}{c}0.183078 \\
\mathbf{5 8}\end{array}$ & $\begin{array}{c}0.421431 \\
59\end{array}$ & $\begin{array}{c}\text { Goal } \\
60\end{array}$ \\
\hline
\end{tabular}

Fig5.Integration of self learning module with decision tree knowledge base.

Figure.5 is a temperature color-coded visualization for the variance in cumulative rewards of neighbors. The numerical value in each state specifies the variance in difference of cumulative rewards of its neighbors when the particular state is blocked. The color of any state is its simple translation of numerical value over hue-scale. The change in color of adjacent states is more significant for identifying hallway regions than the color themselves.

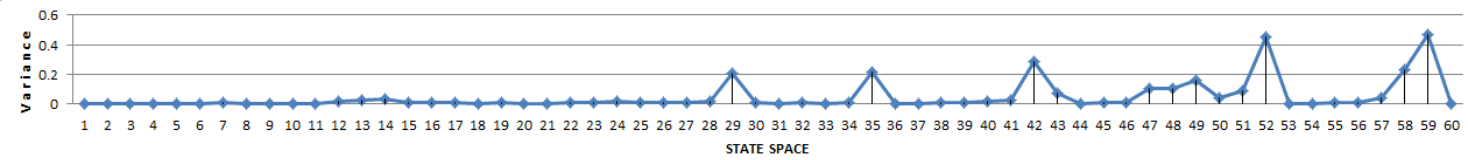

Fig.6 Variance in difference of cumulative rewards of neighboring states upon blocking a particular state. $\mathrm{x}$ -axis considers this variance for each state in entire state space.. 
The graph plot for variance in cumulative rewards presented in Figure.6 is yet another easier interpretation of above color-coded maze.

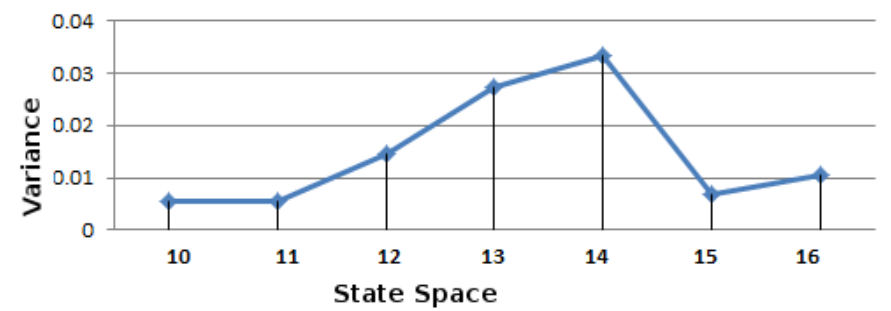

Fig.7 Exaggerated version of Figure.6 over few states of 10:16, highlighting the fluctuation of variance in the hallway region

The fluctuation of local-maxima indicates important states that are correctly identified as hallway regions. An exaggerated version of Figure.6 over the states 10:16 is depicted in Figure.7.

This observation reinforces our positive results verifying the correctness. The above results indicate that hallway regions are found to exhibit a local-maxima property in variance vs. statespace graph.Below we present a few test cases that characterise the general behaviour of scheduler interaction with knowledge base and self-improving module. We also analyse the effectiveness of integrating Static knowledge base and self-learning module by calculating time saved and number of CPU cycles conserved. Programs were verified after executing multiple times with different nice values on Linux System. Their corresponding figures show how the turn-around-time changed as the CPU allotted timeslice of the process changed.

Experiments show that there does not exist any direct evident relation between time slice and CPU utilization performance metrics. Refer Figure. 8 and Figure. 9 plot of TaT vs. timeslice class allotted. Hence it is not a simple linear function which is monotonic in nature. One will have to learn a proper classifier which can learn the pattern and predict optimal timeslice. Below we show the analysis for 900x900 matrix multiplcation and merge sort (input size 3e6). Table.2 shows their new suggested class from knowledge base. For Heapsort (input size 6e5) and Quicksort (input size 1e6) we have only plotted their TaT vs. Timeslice graphs in Figure.8, which is similar in wavy nature as Figure.9. We have omitted explicit calculations to prevent redundancy in paper, as their nature is very similar to previous matrix multiplication.

Effectiveness analysis for Matrix Multiplication with input size of 900x900 random matrix elements.

- Turnaround Time (normal) - $27872216 \mathrm{~ms}$

- Turnaround Time (with KB)- $24905490 \mathrm{~ms}$

- $\quad$ Time saved $=2966726 \mathrm{~ms}$

- Time saved per second - 109879 ms 
- $\quad$ No. of clock cycles saved - 2.4MHz x 109879

- No. of Lower operations saved - 109879 / (pipeline clear + context switch etc.)

(a)

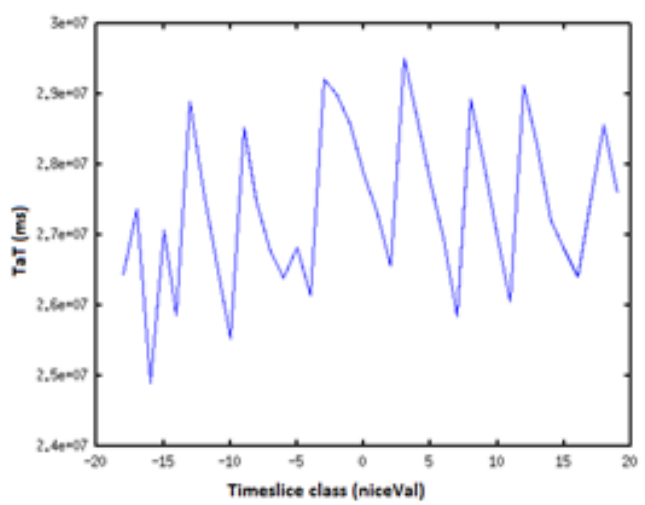

(b)

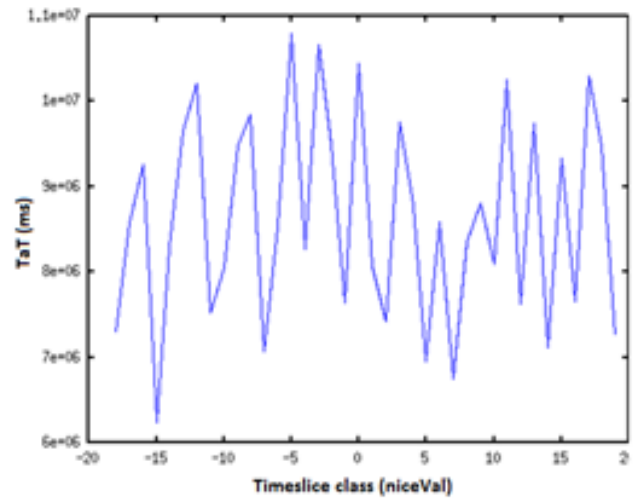

Fig.8 Timeslice class (unnormalized nice values) vs.

Turn around time for (a) Matrix Multiplication and (b)Merge Sort .

\begin{tabular}{|l||l||l|l|}
\hline \multicolumn{2}{|c||}{ Matrix Multiplcation } & \multicolumn{2}{c|}{ Merge Sort } \\
\hline $\begin{array}{l}\text { Turn around time } \\
\text { (microsec) }\end{array}$ & $\begin{array}{l}\text { Timeslice class } \\
\text { suggested }\end{array}$ & $\begin{array}{l}\text { Turn around time } \\
\text { (microsec) }\end{array}$ & $\begin{array}{l}\text { Timeslice class } \\
\text { suggested }\end{array}$ \\
\hline 24905490 & 16 & 6236901 & 15 \\
\hline 25529649 & 10 & 7067141 & 7 \\
\hline 25872445 & 14 & 7305964 & 18 \\
\hline 26151444 & 4 & 7524301 & 11 \\
\hline 26396064 & 6 & 7639436 & 1 \\
\hline 26442902 & 18 & 8055423 & 10 \\
\hline 26577882 & 11 & 8273131 & 4 \\
\hline 26800116 & 7 & 8302461 & 14 \\
\hline 26827546 & 5 & 8537245 & 6 \\
\hline 27080158 & 15 & 8569818 & 17 \\
\hline 27376257 & 17 & 9255897 & 16 \\
\hline 27484162 & 8 & 9483901 & 9 \\
\hline 27643193 & 12 & 9499732 & 2 \\
\hline 28535686 & 9 & 9660585 & 13 \\
\hline 28581739 & 1 & 9844913 & 8 \\
\hline 28900769 & 13 & 10217774 & 12 \\
\hline
\end{tabular}

Table.2 Optimal timeslice-class decisions made by knowledge base module for Matrix multiplication of input 900x900 and Merge sort over input size 3e6 elements. 


\section{Effectiveness analysis for Merge Sort with input size of 3e6 random array elements.}

- $\quad$ TaT (normal) - $10439931 \mathrm{~ms}$ and TaT(with KB)- $6236901 \mathrm{~ms}$

- $\quad$ Time saved $=4203030 \mathrm{~ms}$

- Time saved per second - $382093 \mathrm{~ms}$

- No. of clock cycles saved $-2.4 \mathrm{MHz}$ x 382093

- No. of Lower operations saved - 382093 / (pipeline clear + context switch etc.)

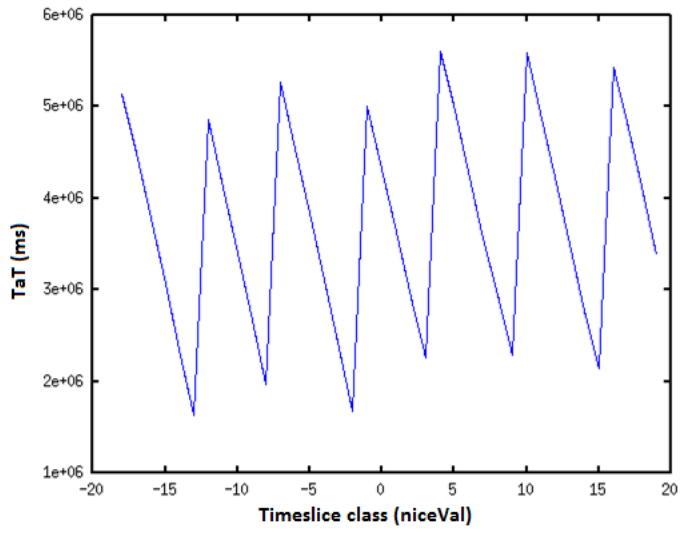

(a)

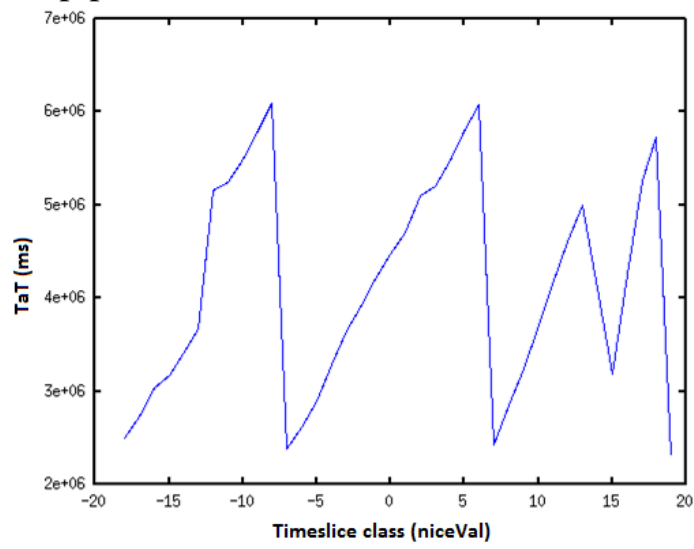

(b)

Fig.9 Timeslice class (unnormalized nice values) vs. Turn around time for (a)Heap Sort, (b)Quick Sort .

\begin{tabular}{|c|c|c|c|c|c|c|c|}
\hline 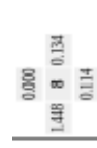 & 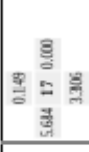 & 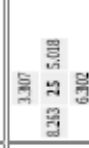 & 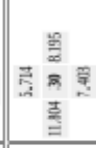 & & $\frac{9}{2}$ & 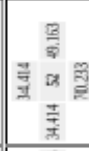 & 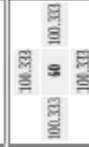 \\
\hline$-\frac{8}{4}$ & 要 & 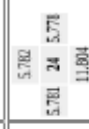 & 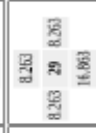 & 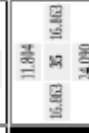 & 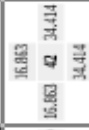 & 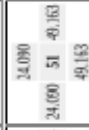 & 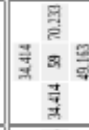 \\
\hline 6 & 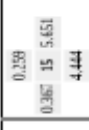 & 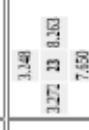 & 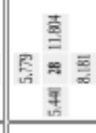 & & $f^{\prime}$ & 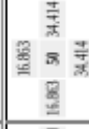 & 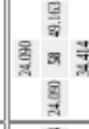 \\
\hline$=$ & 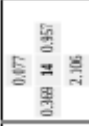 & 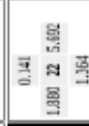 & 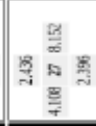 & & 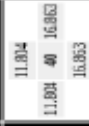 & 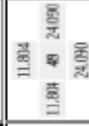 & 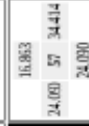 \\
\hline & $\begin{array}{l}\text { s } \\
\approx\end{array}$ & & & & & & \\
\hline 要 & 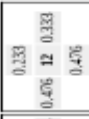 & 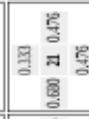 & & 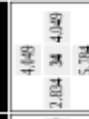 & 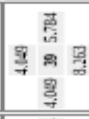 & 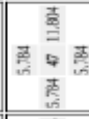 & 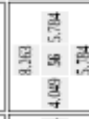 \\
\hline - & 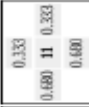 & 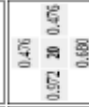 & & 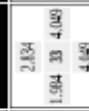 & 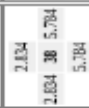 & 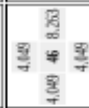 & 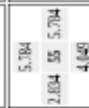 \\
\hline $\mathrm{p}^{\mathrm{p}}$ & 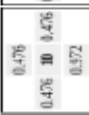 & 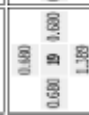 & 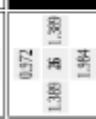 & 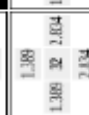 & 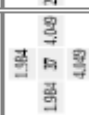 & 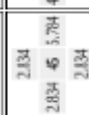 & $\frac{3}{3}$ \\
\hline 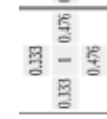 & 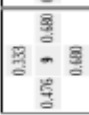 & 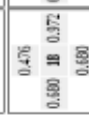 & & 总 & 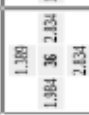 & 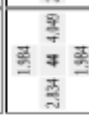 & 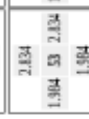 \\
\hline
\end{tabular}

Fig.10 Values learned from iterative Q-learning steps for each cell state in Fig.3 after blocking state 13. Values represent the cumulative rewarding of taking action in that particular direction. 


\section{Effectiveness analysis for Heap Sort with input size of $6 \mathrm{e} 5$ random array elements.}

- $\quad$ Turnaround Time (normal) - $4320086 \mathrm{~ms}$

- Turnaround Time (with KB)- $1678159 \mathrm{~ms}$

- $\quad$ Time saved $=2641927 \mathrm{~ms}$

- $\quad$ Time saved per second $-880642 \mathrm{~ms}$

- No. of clock cycles saved - $2.4 \mathrm{MHz}$ x 880642

- No. of Lower operations saved - 880642 / (pipeline clear + context switch etc.)

\section{System essential statistics}

- User time (seconds): 0.81

- System time (seconds): 0.00

- Percent of CPU this job got: $99 \%$

- Elapsed (wall clock) time (h:mm:ss or m:ss): 0:00.82

In the Fig.10, we observe the influence of blocking a random state (state 13 in this case) and report the behaviour of neighboring states. As 13 lies in hallway region, it causes above average change in cumulative rewards of 12 and 14, as compared to values in Fig.5.

\section{DISCUSSION AND CONCLUSIONS}

It is apparent that states near hallway region show sudden fluctuation in variance of rewards upon blocking them. An interesting fact to be noticed here is that the states adjacent to hallway (like 29, 47) show greater change than the hallway state itself (like 35, 48). Our algorithm suggests that both the ends of the hallway should be perceived to be more important than the hallway itself. We humans would consider mid-hallway as a natural option for an agent, but for what is intuitive to humans may not necessarily be the best optimized solution for machines. Another side observation is the gradual increase in numerical value of variance of cumulative reward level for states that are nearer to the goal-state. This is expected, as the states achieve more importance in being closer to the goal state, which causes numerical bias of rewards as we approach the goal. As Q-learning equation suggests, one extra step towards the goal will deduce the optimal policy faster. Hence it is sensible for agent to cross over the hallway, before termination, within the same option.

Further from the scheduler results we can observe that the turnaround time can be optimized by reducing redundant context switches and also reducing the additional lower level register swaps, pipeline clearances etc. This in turn saves the CPU cycles that are valuable resource for runtime execution of subsequent jobs. A self-learning module proposed here has the potential of constantly improving with more experiences and is provided over a knowledge base to prevent the problem of cold-start. We have showed the non-intuitive irregularity between decreasing turnaround time and increasing time slice by wave-pattern of TaT vs. class of time.

As one may observe, the above procedure is computationally intensive and would be expensive in case of large state-space. But identification of optimal option subgoals motivates for future work on approximation algorithms that stochastically arrive at the same solution with good precision. We are also currently investigating ways to address the problem of infinite horizon in reinforcement learning, as the scheduler may run for infinite amount of time (or very large time unit) and scores rewards just for the sake of its existence. 


\section{REFERENCES}

1. Warren Smith, Valerie Taylor, Ian Foster, Predicting Application Run-Times Using Historical Information”, Job Scheduling Strategies for Parallel Processing, IPPS/SPDP‘98 Workshop, March, 1998.

2. Jonathan M Eastep, "Smart data structures: An online ML approach to multicore Data structure", IEEE Real-Time and Embedded Technology and Applications Symposium 2011.

3. M. John Calandrino, documentation for the main source code for LinSched and author the linux_linsched files LINK: http://www.cs.unc.edu/ jmc/linsched/

4. D. Vengerov, A reinforcement learning approach to dynamic resource scheduling allocation, Engineering Applications of Artificial Intelligence, vol. 20, no 3, p. 383-390, Elsevier, 2007.

5. Richard Gibbons, A Historical Application Profiler for Use by Parallel Schedulers, Lecture Notes on Computer Science, Volume : 1297, pp: 58-75, 1997.

6. Richard S. Sutton and Andrew G. Barto., Reinforcement Learning: An Introduction. A Bradford Book. The MIT Press Cambridge, Massachusetts London, England.

7. Atul Negi, Kishore Kumar. P, UOHYD, Applying machine learning techniques to improve Linux process scheduling 2010.

8. Internals of Linux kernel and documentation for interface modules LINK: http://www.faqs.org/docs/kernel_2_4/lki-2.html

9. D. Vengerov, A reinforcement learning framework for utility-based scheduling in resourceconstrained systems, Future Generation Compute Systems, vol. 25, p. 728-736 Elsevier, 2009.

10. Surkanya Suranauwarat, Hide Taniguchi, The Design, Implementation and Initial Evaluation of An Advanced Knowledge-based Process Scheduler, ACM SIGOPS Operating Systems Review, volume: 35, pp: 61-81, October, 2001.

11. Documentation for IBM project for real scheduler simulator in User space LINK: http://www.ibm.com/developerworks/library/l-linux-schedulersimulator/

12. Tong Li, Jessica C. Young, John M. Calandrino, Dan P. Baumberger, and Scott Hahn, LinSched: The Linux Scheduler Simulator Research Paper by Systems Technology Lab Intel Corporation, 2008

13. McGovern, A., Moss, E., and Barto, A. G. (2002). Building a basic block instruction scheduler with reinforcement learning and rollouts. Machine Learning, 49(2/3):141- 160.

14. Martin Stolle and Doina Precup, Learning Options in Reinforcement Learning Springer-Verlag Berlin Heidelberg 2002

15. Danie P. Bovet, Marc, Understanding the Linux Kernel, 2nd ed, O‘ Reilly and Associates, Dec., 2002.

16. Modern Operation System Scheduler Simulator, development work for simulating LINK: http://www.ontko.com/moss/

17. Andrew Marks, A Dynamically Adaptive CPU Scheduler, Department of Computer Science, Santa Clara University, pp :5- 9, June, 2003.

18. Prakhar Ojha, Thota Siddhartha R, Vani M, Tahilianni Mohit, Learning Scheduler Parameters for Adaptive Preemption, 2015, National Institute of Technology Karnataka, pp: 148-162, SCAI'15 ICAITA.

19. X. Ding, Y.-t. LI, and S. Chuan. Autonomic discovery of subgoals in hierarchical reinforcement learning. The Journal of China Universities of Posts and Telecommunications, 21(5):94-104, 2014

20. D. R. H. Lathropb Thomas G. Solving the multiple-instance problem with axis-parallel rectangles.

21. A. McGovern. acquire-macros: An algorithm for automatically learning macro-actions. In NIPS98 Workshop on Abstraction and Hierarchy in Reinforcement Learning, 1998.

22. A. McGovern, R. S. Sutton, and A. H. Fagg. Roles of macro-actions in accelerating reinforcement learning. In Grace Hopper celebration of women in computing, volume 1317, 1997.

23. R. B. Myerson. Conference structures and fair allocation rules. International Journal of Game Theory, 9(3):169-182, 1980.

24. D. Precup. Temporal abstraction in reinforcement learning. 2000.

25. M. Stolle and D. Precup. Learning options in reinforcement learning. In SARA, pages 212-223. Springer, 2002.

26. R. S. Sutton and A. G. Barto. Reinforcement learning: An introduction, volume 1. MIT press Cambridge, 1998.

27. R. S. Sutton, D. Precup, and S. Singh. Between mdps and semi-mdps: A framework for temporal abstraction in reinforcement learning. Artificial intelligence, 112(1):181-211, 1999. 


\section{Authors}

Prakhar Ojha, student of the Department of Computer Science Engineering at National Institute of Technology Karnataka Surathkal, India. His areas of interest are Artificial Intelligence, Reinforcement Learning and Application of knowledge bases for smart decision making.

Siddhartha R Thota, student of the Department of Information Technology at National Institute of Technology Karnataka Surathkal, India. His areas of interest are Machine Learning, Natural language processing and hidden markov model based Speech processing.

Vani M is a Associate Professor in the Computer Science Engineering Department of NITK. She has over 18 years of teaching experience. Her research interest includes Data Structures and Algorithms, Algorithmic graph theory, Operating systems and Algorithms for wireless sensor networks.

Mohit P Tahiliani is a Assistant Professor in the Computer Science Engineering Department of NITK. His research interest includes Named Data Networks, TCP Congestion Control, Bufferbloat, Active Queue Management (AQM) mechanisms and Routing Protocol Design and Engineering.
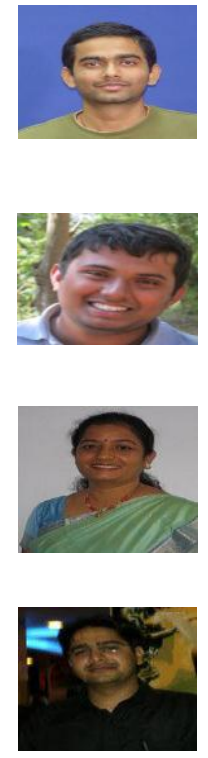